\title{
Nonalcoholic Fatty Liver Disease and its association with Insulin Resistance: A Study from Bangladeshi Newly Diagnosed Impaired Glucose Tolerance Subjects
}

Israt $\mathrm{AH}$ and Liaquat $\mathrm{A}^{*}$

Department of Biochemistry and Cell Biology, Bangladesh University of Health Sciences, Dhaka, Bangladesh

\begin{abstract}
Background: Nonalcoholic fatty liver disease (NAFLD) is a clinicopathological condition strongly associated with obesity, type 2 diabetes, and metabolic syndrome. NAFLD is more prevalent in T2DM subjects but in the prediabetic state, particularly in impaired glucose tolerance (IGT) very, limited data are elucidated. The present study is aimed to examine the association of insulin resistance with NAFLD in subjects with newly diagnosed IGT.

Methods: We studied 74 purposively recruited IGT [M/F, 34/40; age (ranges) in years, (25-64)] subjects whose NAFLD was confirmed by ultrasound assessment of the liver. IGT was diagnosed by $75 \mathrm{~g}$ OGTT as per WHO Group Study criteria. Serum glucose was measured by glucose-oxidase method. Serum insulin was measured by ELISA technique. Insulin resistance (HOMA-IR) was calculated by homeostasis model assessment (HOMA).

Results: Compared to the without NAFLD counterparts, NAFLD subjects showed significantly higher levels of fasting serum insulin (17.26 \pm 8.49 vs. $13.38 \pm 4.40, p=0.015)$, postprandial serum insulin $(90.06 \pm 42.23$ vs. $69.87 \pm$ $38.10, p=0.034)$ and $\mathrm{HOMA}-\mathrm{IR}(2.20 \pm 1.0$ vs. $1.73 \pm 0.55, \mathrm{p}=0.012)$ as well as significantly lower levels of HOMA\%S $(52.13 \pm 20$ vs. $63.92 \pm 25.44, p=0.029)$. On multiple linear regression analysis, the fatty liver group showed significant positive association with HOMA-IR $(\beta=0.270, p=0.020)$ after adjusting the effects of major confounders of sex, percent body fat (\%BF) and body mass index (BMI). On binary logistic regression analysis, HOMA-IR was found to be a significant determinant of NAFLD $(O R=2.679,95 \% \mathrm{Cl}: 1.079-6.652, p=0.034)$ after adjusting the effects of $\% \mathrm{BF}, \mathrm{BMI}$, and triglyceride (TG)
\end{abstract}

Conclusions: High proportions (about forty seven percent) of IGT subjects are more likely to develop NAFLD. Altered glucose metabolism and fatty liver are associated with each other where insulin resistance plays linking role between their associations.

Keywords: Insulin resistance; Nonalcoholic fatty liver disease; Impaired glucose tolerance

Abbreviations: NAFLD: Nonalcoholic Fatty Liver Disease; IGT: Impaired Glucose Tolerance; T2DM: Type 2 Diabetes Mellitus; MS: Metabolic Syndrome; NASH: Nonalcoholic Steatohepatitis; $\mathrm{Hba}_{1 \mathrm{c}}$ : Glycosylated Hemoglobin; HPLC: High Performance Liquid Chromatography; ELISA: Enzyme-Linked Immunosorbent Assay; ROS: Reactive Oxygen Species; BMI: Body Mass Index; WC: Waist Circumference; HC; Hip Circumference; WHR: Waist To Hip Ratio; \% BF: Percent Body Fat; SBP: Systolic Blood Pressure; DBP: Diastolic Blood Pressure; OGTT: Oral Glucose Tolerance Test; FSG: Fasting Serum Glucose; PPSG: Postprandial Serum Glucose; Hba Glycosylated Hemoglobin; TC: Total Cholesterol; TG: Triglyceride; HDL-C: High Density Lipoprotein-Cholesterol; LDL-C: Low Density Lipoprotein-Cholesterol; ALT: Alanine Transaminase; AST; Aspartate Transaminase; T-GT: Gamma Glutamate Transaminase; ALP: Alkaline Phosphatase; IL-6: Interleukin-6; ELISA: Enzyme Linked Immunosorbent Assay; FSI: Fasting Serum Insulin; PPSI: Postprandial Serum Insulin; HOMA\%S: Insulin Sensitivity Assessed By Homeostasis Model Assessment; HOMA-IR: Homeostasis Model Assessment Insulin Resistance

\section{Introduction}

Nonalcoholic fatty liver disease (NAFLD) is a hepatic disease encompasses a spectrum of simple steatosis, nonalcoholic steatohepatitis (NASH), to cirrhosis. In the general population, the disease has a prevalence of $10-24 \%$ [1]. The pathogenesis of NAFLD appears to involve a two-hit hypothesis [2]. The first hit is the accumulation of excessive free fatty acid (FFA) within the hepatocytes due to impaired insulin action following in a secondary event of oxidative stress, resulting NASH. Hyperinsulinemia induces insulin resistant condition by inhibiting the beta oxidation of FFA which ultimately reesterified into triacylglyceride (TAG) and deposited into the liver. Increased lipotoxicity leads to a condition of mitochondrial dysfunction thereby releasing the reactive oxygen species (ROS). The free radicals released by ROS causes inflammation by activating the inflammatory cytokines leading to hepatic injury [3].

Prior epidemiological studies demonstrates a higher prevalence of NAFLD among the T2DM subjects which is likely 2.6 fold increase risk of NASH who are hyperglycemic [4]. However, the putative mechanism relating the development of NAFLD among the hyperglycemic subjects remains unclear. It is argued that whether insulin resistance is a cause

${ }^{*}$ Corresponding author: Liaquat A, Department of Biochemistry and Cell Biology, Bangladesh University of Health Sciences, Dhaka, Bangladesh, Tel: +88-029010654; Fax: +88-02-8055312; E-mail: vc@buhs.edu.bd

Received June 21, 2016; Accepted July 12, 2016; Published July 18, 2016

Citation: Israt AH, Liaquat A (2016) Nonalcoholic Fatty Liver Disease and its association with Insulin Resistance: A Study from Bangladeshi Newly Diagnosed Impaired Glucose Tolerance Subjects. J Diabetes Metab 7: 688. doi: 10.4172/21556156.1000688

Copyright: $\odot 2016$ Israt $\mathrm{AH}$, et al. This is an open-access article distributed unde the terms of the Creative Commons Attribution License, which permits unrestricted use, distribution, and reproduction in any medium, provided the original author and source are credited. 
Citation: Israt AH, Liaquat A (2016) Nonalcoholic Fatty Liver Disease and its association with Insulin Resistance: A Study from Bangladeshi Newly Diagnosed Impaired Glucose Tolerance Subjects. J Diabetes Metab 7: 688. doi: 10.4172/2155-6156.1000688

Page 2 of 5

or consequence of NAFLD which strongly correlated with insulin resistant states such as obesity, MS, and T2DM. NAFLD is a metabolic disorder and that insulin resistance plays a key role in its genesis. A number of clinical studies show a strong association of insulin resistance with NAFLD among nonobese healthy subjects $[5,6]$.

Although the association of insulin resistance in diabetic NAFLD subjects is well known [6], its role in insulin resistant state, particularly in IGT subjects with NAFLD has been less well established. Since subjects with IGT are the role model of insulin resistance which also the basic defect in NAFLD, it is necessary to investigate this group to explore this relationship.

\section{Materials and Methods}

\section{Subject's selection}

A case-control study was conducted in the 74 (seventy four) IGT subjects attending the BIHS Hospital, Darussalam, Dhaka, Bangladesh, in the period from March to October 2012. Subjects were excluded who had acute and chronic hepatic, cardiac, renal, respiratory diseases, stroke, type 1 diabetes, those taking drugs that significantly affect the glucose and lipid metabolism, antihypertensive, and pregnant subjects. IGT was diagnosed by WHO Group Study criteria following a 2 sample OGTT [7]. The study was approved by the ethical review committee of Bangladesh Diabetic Association (BADAS). Ref no: BADASERC/13/00106. Each participant gave written informed consent prior to study inclusion. Anthropometric measurements including height, weight, waist and hip circumference, systolic and diastolic blood pressures (SBP and DBP) were measured by standard procedures. Body mass index (BMI) was calculated as weight $(\mathrm{kg})$ divided by height $(\mathrm{m})$ squared.

\section{Laboratory measurements}

Serum glucose, both at fasting and following ingestion of $75 \mathrm{~g}$ of glucose, serum lipid profile (total cholesterol, triglycerides and HDL-c) and liver function enzymes like aspartate transaminase (AST), alanine transaminase (ALT), alkaline phosphatase (ALP) and gamma glutamyl transaminase $(\gamma-\mathrm{GT})$ were measured by enzymatic-colorimetric method using an automated analyzer (Dimension ${ }^{\circledR}$ clinical chemistry system, Siemens Healthcare Diagnostics Inc. USA). Low-density lipoprotein cholesterol (LDL-c) was calculated by the Friedewald equation [8]. Serum insulin was measured by an enzyme linked immune assay (ELISA) method (DRG-International, Germany) whose intra-assay and inter-assay CVs were $6.3 \%$ and $4.25 \%$ respectively when specific performance characteristics were assessed. Insulin resistance was determined by the homeostasis model assessment insulin resistance (HOMA-IR) and calculated according to the formula: Fasting serum insulin $(\mu \mathrm{IU} / \mathrm{ml}) \times$ fasting serum glucose $(\mathrm{mmol} / \mathrm{l}) / 22.5[9]$.

\section{NAFLD evaluation}

Ultrasonography examination was performed by a well trained physician who was unaware the purpose of the study using a 3.5 $\mathrm{MHz}$ linear transducer (Philips Ultrasound-Ay-MNT-15 TTK, HDI-4000, Netherland) sonography machine. Grades of fatty liver were recorded as: Grade 0 (normal), Grade 1 (mild), Grade 2 (moderate), and Grade 3 (severe) [10].

\section{Statistical analysis}

Statistical analysis was performed using the SPSS software package version 15.0 for Windows (SPSS Inc., Chicago, IL, USA).
Differences between the groups were assessed by student's t-test and the association of two parameters was explored by univariate and multivariate analysis as appropriate. Multiple linear regression analysis was done to see the association of HOMA-IR with the fatty liver group considering HOMA-IR as the dependable variable and sex, \%BF, BMI, and fatty liver group as the independent variables. Multivariate logistic regression analysis was carried out considering groups (NAFLD and without NAFLD as reference) as the dependent variable and $\% \mathrm{BF}, \mathrm{BMI}$, TG and HOMA-IR as independent variables to assess significant predictors and adjusted odds ratio estimated by controlling the above significant predictors of NAFLD. All of the reported p values (2-tailed test) less than 0.05 were considered to be statistically significant.

\section{Results}

Table 1 shows the general characteristics of the IGT subjects. Of the total 74 IGT subjects, male and the female was 34 (45.9\%) and $40(54.1 \%)$, BMI and \%BF was $25.71 \pm 3.98$ and $29.84 \pm 7.47$

\begin{tabular}{|c|c|}
\hline Variables & $\begin{array}{l}\text { IGT Subjects } \\
\quad(n=74)\end{array}$ \\
\hline \multicolumn{2}{|l|}{ Gender (n, \%) } \\
\hline Males & $34(45.9)$ \\
\hline Females & $40(54.1)$ \\
\hline Age (years) & $42.15 \pm 9.59$ \\
\hline $\mathrm{BMI}\left(\mathrm{kg} / \mathrm{m}^{2}\right)$ & $25.71 \pm 3.98$ \\
\hline WC (cm) & $90.03 \pm 8.06$ \\
\hline $\mathrm{HC}(\mathrm{cm})$ & $95.27 \pm 7.91$ \\
\hline WHR & $0.94 \pm 0.04$ \\
\hline$\% \mathrm{BF}$ & $29.84 \pm 7.47$ \\
\hline SBP $(\mathrm{mmHg})$ & $126 \pm 24$ \\
\hline $\mathrm{DBP}(\mathrm{mmHg})$ & $84 \pm 20$ \\
\hline Without NAFLD (n, \%) & $39(52.7)$ \\
\hline $\operatorname{NAFLD~}(\mathrm{n}, \%)$ & $35(47.3)$ \\
\hline \multicolumn{2}{|c|}{ Grades of fatty liver (n, \%) } \\
\hline Grade 0 & $38(51.4)$ \\
\hline Grade 1 & $25(33.8)$ \\
\hline Grade 2 & $9(12.2)$ \\
\hline Grade 3 & $2(2.7)$ \\
\hline $\mathrm{FSG}(\mathrm{mmol} / \mathrm{l})$ & $5.22 \pm 0.22$ \\
\hline PPSG (mmol/l) & $8.94 \pm 0.99$ \\
\hline $\mathrm{HbA}_{1 \mathrm{C}}(\%)$ & $5.77 \pm 0.61$ \\
\hline TC (mg/dl) & $194 \pm 34$ \\
\hline TG (mg/dl) & $158 \pm 61$ \\
\hline HDL-c (mg/dl) & $38 \pm 8$ \\
\hline LDL-c (mg/dl) & $186 \pm 41$ \\
\hline $\operatorname{ALT}(\mathrm{U} / \mathrm{L})$ & $36.22 \pm 19.02$ \\
\hline V-GT (U/L) & $33.46 \pm 15.45$ \\
\hline AST (U/L) & $97.96 \pm 22.86$ \\
\hline $\operatorname{ALP}(\mathrm{U} / \mathrm{L})$ & $31.72 \pm 12.83$ \\
\hline $\mathrm{FSI}(\mu \mathrm{lU} / \mathrm{ml})$ & $15.22 \pm 6.89$ \\
\hline PPSI $(\mu \mathrm{lU} / \mathrm{ml})$ & $79.42 \pm 41.10$ \\
\hline HOMA\%S & $58.34 \pm 23.63$ \\
\hline HOMA-IR & $1.95 \pm 0.82$ \\
\hline
\end{tabular}

Results are given as mean \pm standard deviation or number of subjects (\%); Level of significance was calculated by Students't' test; $n=$ number of subjects; IGT: Impaired Glucose Tolerance; HOMA\%S: Insulin Sensitivity assessed by Homeostasis Model Assessment; HOMA-IR; Homeostasis Model Assessment Insulin Resistance.

Table 1: General characteristics of the study subjects. 
Citation: Israt AH, Liaquat A (2016) Nonalcoholic Fatty Liver Disease and its association with Insulin Resistance: A Study from Bangladeshi Newly Diagnosed Impaired Glucose Tolerance Subjects. J Diabetes Metab 7: 688. doi: 10.4172/2155-6156.1000688

Page 3 of 5

respectively. After liver ultrasonography, the without NAFLD and NAFLD groups were $39(52.7 \%)$ and $35(47.3 \%)$ respectively of where, grade 0 , grade 1 , grade 2 and grade 3 were $38(51.4 \%), 25$ (33.8\%), 9 (12.2\%) and $2(2.7 \%)$ respectively.

Table 2 shows the anthropometric, clinical and biochemical characteristics of without and with NAFLD subjects. There was $17(43.6 \%)$ male and $22(56.4 \%)$ female in the without NAFLD group and there was $17(48.6 \%)$ male and $18(51.4 \%)$ female in the NAFLD group. The grade 0 , grade 1 and grade 2 were $33(84.6 \%)$, $4(10.3 \%)$, and $2(5.1 \%)$ in the without NAFLD group while this values along with grade 3 in the NAFLD group were $5(14.3 \%)$, $21(60 \%), 7(20 \%)$ and $2(5.7 \%)$ respectively. Compared to their without NAFLD counterparts, NAFLD subjects had significantly higher fasting serum insulin $(17.26 \pm 8.49$ vs. $13.38 \pm 4.40 \mu \mathrm{IU} /$ $\mathrm{ml}, \mathrm{p}=0.015)$, postprandial serum insulin $(69.87 \pm 38.10 \mathrm{vs.} 90.06 \pm$ $42.23 \mu \mathrm{IU} / \mathrm{ml}, \mathrm{p}=0.034)$ and HOMA-IR $(2.20 \pm 1.0$ vs. $1.73 \pm 0.55$, $\mathrm{p}=0.029)$ respectively, however, they had significantly lower levels of HOMA\%S (52.13 \pm 20 vs. $63.92 \pm 25.44, \mathrm{p}=0.012)$.

To get a deeper understanding of the relationship between insulin resistance and NAFLD, the study subjects were stratified into insulin sensitive (HOMA-IR $\leq 2.0$ ) and insulin resistant (HOMAIR $\geq 2.0$ ) groups. The frequency of insulin sensitive and resistance subjects were $71.8 \%(n=28)$ and $28.2 \%(n=11)$ in the group of without NAFLD whereas, these values in the NAFLD group were $42.9 \%(n=15)$ and $57.1 \%(n=20)$ respectively. With increasing the prevalence of NAFLD, the quartiles of HOMA-IR also increased progressively and the increment is higher in the NAFLD group compared to their without NAFLD counterparts (Figure 1).

Table 3 shows the bivariate Pearson's correlation analyses of HOMA-IR with clinical and biochemical variables in the NAFLD subjects. HOMA-IR showed significant positive correlation with $\operatorname{sex}(\mathrm{r}=0.338, \mathrm{p}=0.047)$, WC $(\mathrm{r}=0.347, \mathrm{p}=0.041)$, WHR $(\mathrm{r}=0.357$, $\mathrm{p}=0.042), \% \mathrm{BF}(\mathrm{r}=0.357, \mathrm{p}=0.035), \mathrm{BMI}(\mathrm{r}=0.345, \mathrm{p}=0.042)$ and GGT $(r=0.369, \mathrm{p}=0.029)$ respectively.

Table 4 shows the multiple linear regression analysis using HOMA-IR as dependent variable and sex, \%BF, BMI and the group as independent variables. HOMA-IR showed significant positive association with the group $(\beta=0.270, p=0.020)$ after adjusting the effects of major confounding variables of sex, $\% \mathrm{BF}$ and $\mathrm{BMI}$ respectively.

Table 5 shows the binary logistic regression analysis using NAFLD as dependent variable and \%BF, BMI, TG and HOMA-IR as independent variables. HOMA-IR ( $\mathrm{OR}=2.679,95 \% \mathrm{CI}$ : $1.079-6.652$, $\mathrm{p}=0.034$ ) was found to be significant determinant of NAFLD after adjusting the effects of major confounding variables of $\% \mathrm{BF}, \mathrm{BMI}$, and TG respectively.

\section{Discussion}

The present study demonstrates a higher prevalence of NAFLD among the IGT subjects. In a study by Mohan et al. the prevalence of NAFLD in subjects with isolated IGT was (32.4\%) which is in accordance with our study where we found the prevalence of NAFLD is about $45.2 \%$ in the isolated IGT subjects [11]. We performed the ultrasonography imaging for the histological analysis of the liver because of its non-invasive, cost effective and readily available tool in diagnosing NAFLD rather than the liver biopsy (gold standard method).

\begin{tabular}{|c|c|c|c|}
\hline Variables & $\begin{array}{l}\text { IGT without } \\
\text { NAFLD } \\
(n=39)\end{array}$ & $\begin{array}{c}\text { IGT with NAFLD } \\
(n=35)\end{array}$ & t/p-value \\
\hline \multicolumn{4}{|c|}{ Gender (n, \%) } \\
\hline Males & $17(43.6)$ & $17(48.6)$ & \\
\hline Females & $22(56.4)$ & $18(51.4)$ & \\
\hline Age (years) & $40.62 \pm 9.21$ & $43.86 \pm 9.84$ & $-1.462 / 0.148$ \\
\hline BMI $\left(\mathrm{kg} / \mathrm{m}^{2}\right)$ & $24.92 \pm 3.97$ & $26.59 \pm 3.84$ & $-1.823 / 0.072$ \\
\hline WC $(\mathrm{cm})$ & $88.67 \pm 7.57$ & $91.54 \pm 8.42$ & $-1.546 / 0.126$ \\
\hline $\mathrm{HC}(\mathrm{cm})$ & $94.51 \pm 7.24$ & $96.11 \pm 8.62$ & $-0.868 / 0.388$ \\
\hline WHR & $0.93 \pm 0.04$ & $0.95 \pm 0.05$ & $-1.380 / 0.172$ \\
\hline$\% \mathrm{BF}$ & $29.39 \pm 7.20$ & $30.34 \pm 7.83$ & $-0.545 / 0.587$ \\
\hline $\mathrm{SBP}(\mathrm{mmHg})$ & $123 \pm 20$ & $128 \pm 29$ & $-0.860 / 0.392$ \\
\hline $\mathrm{DBP}(\mathrm{mmHg})$ & $81 \pm 16$ & $88 \pm 23$ & $-1.496 / 0.139$ \\
\hline \multicolumn{4}{|l|}{$\begin{array}{l}\text { Grades of fatty liver } \\
\qquad(\mathrm{n}, \%)\end{array}$} \\
\hline Grade 0 & $33(84.6)$ & $5(14.3)$ & - \\
\hline Grade 1 & $4(10.3)$ & $21(60)$ & - \\
\hline Grade 2 & $2(5.1)$ & $7(20)$ & - \\
\hline Grade 3 & - & $2(5.7)$ & - \\
\hline FSG (mmol/l) & $5.20 \pm 0.23$ & $5.23 \pm 0.21$ & $-0.503 / 0.616$ \\
\hline PPSG (mmol/l) & $8.84 \pm 0.96$ & $9.06 \pm 1.01$ & $-0.951 / 0.345$ \\
\hline $\mathrm{HbA}_{1 \mathrm{C}}(\%)$ & $5.75 \pm 0.70$ & $5.79 \pm 0.52$ & $-0.243 / 0.808$ \\
\hline $\mathrm{TC}(\mathrm{mg} / \mathrm{dl})$ & $191 \pm 34$ & $198 \pm 33$ & $-0.902 / 0.370$ \\
\hline TG (mg/dl) & $154 \pm 59$ & $162 \pm 65$ & $-0.563 / 0.575$ \\
\hline HDL-c (mg/dl) & $39 \pm 8$ & $36 \pm 7$ & $1.373 / 0.174$ \\
\hline LDL-c (mg/dl) & $187 \pm 43$ & $184 \pm 38$ & $0.262 / 0.794$ \\
\hline ALT (U/L) & $35.41 \pm 19.60$ & $37.11 \pm 18.58$ & $-0.383 / 0.703$ \\
\hline $\mathrm{Y}-\mathrm{GT}(\mathrm{U} / \mathrm{L})$ & $31.49 \pm 16.02$ & $35.66 \pm 14.71$ & $-1.161 / 0.249$ \\
\hline AST (U/L) & $30.15 \pm 13.34$ & $33.46 \pm 12.19$ & $-1.107 / 0.272$ \\
\hline $\operatorname{ALP}(\mathrm{U} / \mathrm{L})$ & $100.31 \pm 23.56$ & $95.34 \pm 22.09$ & $0.932 / 0.355$ \\
\hline $\mathrm{FSI}(\mu \mathrm{lU} / \mathrm{ml})$ & $13.38 \pm 4.40$ & $17.26 \pm 8.49$ & $-2.498 / 0.015$ \\
\hline PPSI ( $\mu \mathrm{IU} / \mathrm{ml})$ & $69.87 \pm 38.10$ & $90.06 \pm 42.23$ & $-2.161 / 0.034$ \\
\hline HOMA\%S & $63.92 \pm 25.44$ & $52.13 \pm 20$ & $2.228 / 0.029$ \\
\hline HOMA-IR & $1.73 \pm 0.55$ & $2.20 \pm 1.0$ & $-2.563 / 0.012$ \\
\hline
\end{tabular}

Results are given as mean \pm standard deviation or number of subjects (\%); Level of significance was calculated by Students't' test; $n=$ number of subjects IGT: Impaired Glucose Tolerance; NAFLD: Nonalcoholic Fatty Liver Disease HOMA\%S: Insulin Sensitivity assessed by Homeostasis Model Assessment; HOMA-IR: Homeostasis Model Assessment Insulin Resistance.

Table 2: Anthropometric, clinical and biochemical characteristics between without and NAFLD subjects.

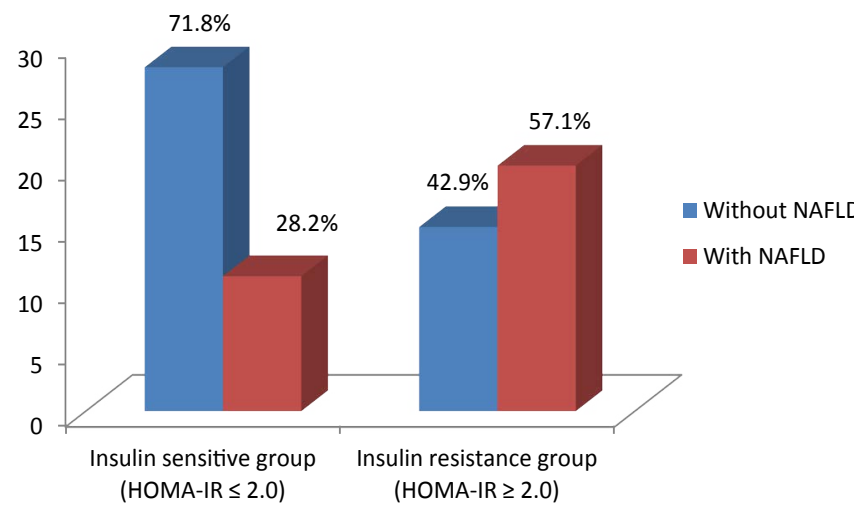

Figure 1: Frequency distribution of fatty liver in the insulin sensitive and resistance group among the IGT subjects. 


\begin{tabular}{|c|c|c|}
\hline \multirow{2}{*}{ Variables } & \multicolumn{2}{|c|}{ HOMA-IR } \\
\hline Gender & r-value & p-value \\
\hline WC $(\mathrm{cm})$ & 0.338 & 0.047 \\
\hline WHR & 0.347 & 0.041 \\
\hline BF $(\%)$ & 0.357 & 0.042 \\
\hline BMI $\left(\mathrm{kg} / \mathrm{m}^{2}\right)$ & 0.357 & 0.035 \\
\hline Y-GT $(\mathrm{U} / \mathrm{L})$ & 0.345 & 0.042 \\
\hline
\end{tabular}

Results are given by Pearson's correlation coefficient $r$ and statistical significance $\mathrm{p}<0.05$; HOMA-IR: homeostasis model assessment insulin resistance.

Table 3: Correlation of HOMA-IR with some significant variables in the total study subjects.

\begin{tabular}{|c|c|c|c|c|c|}
\hline Variables & $\begin{array}{c}\text { Coefficients } \\
(\boldsymbol{\beta})\end{array}$ & t-value & p-value & \multicolumn{2}{|c|}{$95 \%$ C.I. } \\
\cline { 5 - 6 } & - & 0.798 & 0.428 & -0.726 & 1.694 \\
\hline (Constant) & 0.163 & 1.147 & 0.255 & -0.198 & 0.734 \\
\hline Gender & 0.101 & 0.655 & 0.515 & -0.023 & 0.045 \\
\hline BF (\%) & 0.097 & 0.765 & 0.447 & -0.032 & 0.073 \\
\hline BMI (kg/m ${ }^{2}$ ) & 0.023 Uound & Upper Bound \\
\hline Fatty liver group & 0.270 & 2.390 & 0.020 & 0.073 & 0.815 \\
\hline
\end{tabular}

Dependent variable: HOMA-IR; Adjusted $\mathrm{R}^{2}=0.168$; the level of significance at p<0.05; C.I: Confidence Interval; HOMA-IR: Homeostasis Model Assessment Insulin Resistance.

Table 4: Multiple linear regression analysis to explore the association of HOMA-IR with fatty liver group (NAFLD vs. without NAFLD as reference) after adjusting the effects of major confounders.

\begin{tabular}{|c|c|c|c|c|c|c|}
\hline & & & & \multirow{2}{*}{} & & \multicolumn{2}{|c|}{$95 \%$ C.I. } \\
\cline { 5 - 7 } Variables & Coefficient & S. E. & p-value & $\begin{array}{l}\text { Odds } \\
\text { Ratio }\end{array}$ & $\begin{array}{l}\text { Lower } \\
\text { Bound }\end{array}$ & $\begin{array}{c}\text { Upper } \\
\text { Bound }\end{array}$ \\
\hline BF $(\%)$ & -0.033 & 0.039 & 0.397 & 0.967 & 0.896 & 1.045 \\
\hline $\mathrm{BMI}\left(\mathrm{kg} / \mathrm{m}^{2}\right)$ & 0.102 & 0.073 & 0.163 & 1.108 & 0.960 & 1.279 \\
\hline TG $(\mathrm{mg} / \mathrm{dl})$ & 0.003 & 0.004 & 0.509 & 1.003 & 0.995 & 1.011 \\
\hline HOMA-IR & 0.985 & 0.464 & 0.034 & 2.679 & 1.079 & 6.652 \\
\hline Constant & -4.086 & 1.889 & 0.030 & 0.017 & - & - \\
\hline
\end{tabular}

Dependent variable: NAFLD; Adjusted $R^{2}=0.127$; the level of significance at p<0.05; S.E: Standard Error; C.I: Confidence Interval; HOMA-IR: Homeostasis Model Assessment Insulin Resistance.

Table 5: Binary logistic regression analysis taking group (NAFLD vs. without NAFLD as reference) as dependant variable after adjusting the effects of major confounders.

Insulin resistance (IR) is a characteristics feature of NAFLD but it is argued whether it is a cause or consequences. Our study subjects had significantly higher levels of IR in the NAFLD group compared to the non-NAFLD. In a recent study by Petersen et al. [12] showed a higher prevalence of IR among the healthy lean Asian Indians compared to other ethnic groups. They hypothesized for this increased prevalence among this risk group might be associated with increased hepatic triglyceride content and plasma IL-6 concentration. In a previous study, IR was shown to be an independent risk factor for NAFLD in non-diabetic subjects with normal BMI [13].

Our study subjects were overweight $\left(\mathrm{BMI}>25 \mathrm{~kg} / \mathrm{m}^{2}\right)$ that plays a crucial role in the development of NAFLD because body fat distribution is the detrimental risk factor in the progression of fatty liver. Obesity enhances the deposition of increased fat within the hepatocytes leading to a condition of IR, thus decreasing the fatty acid oxidation. During obesity, fat cell released a number of adipocytokines that enhances the inflammation of the liver. Thus IR considered the key factor in the pathogenesis of NAFLD by increasing the rate of lipogenesis thereby promoting the inhibition of lipolysis. However, the relationship between glucose metabolism and NAFLD among the state of prediabetes is not well known. In our study, we found a positive correlation of BMI with IR among the NAFLD subjects. This is in line with a recent case-control study by $\mathrm{Ha}$ et al. showed abdominal fat more susceptible to NAFLD among the non-obese subjects [14].

The IR of the NAFLD subjects may have reported from hyperinsulinemia among the IGT subjects. In our study we found significantly higher levels of postprandial serum insulin in NAFLD subjects compared to the controls. During hyperinsulinemic condition, there is hyper secretion of insulin from the pancreas which ultimately decreases the disposal of hepatic insulin to the peripheral tissues. Manchanayake et al. showed a relationship between postprandial hyperinsulinemia and severity of NAFLD among the hyperglycemic subjects. Since NAFLD itself is an insulin resistant condition and our study subjects was glucose intolerant which also role model of IR. It has been suggested that genetic factors that reduce insulin sensitivity and increase triacylglycerol levels may be responsible for the development of IR among the hyperglycemic subjects [15].

Our study supports the association between IR and NAFLD as represented by the fact that all the subjects studied are glucose intolerant. The present study showed a significantly higher level of fasting insulin, IR and a significantly lower level of HOMA\%S in IGT subjects with NAFLD. Prior clinical studies showed the relationship between IR and NAFLD in subjects with diabetes [16-19] however, this is the first study showing the independent association of IR with NAFLD in the IGT subjects. In order to get deeper understanding the relationship between HOMA-IR and NAFLD, all subjects were segregated into two groups according to their IR quartiles. With increasing the prevalence of NAFLD, the levels of IR also increased among the study subjects. This is in agreement with previous studies which suggest that IR plays a significant role in the progression of the disorder $[13,18]$.

Our binary logistic regression analysis showed a significant positive association of IR with NAFLD after adjusting the potential confounders. In a review finding by Kristina et al. reported IR as a pathogenic risk factor in the development of NAFLD providing a strong association with obesity, T2DM and MS [4]. Bhat et al. demonstrates IR as a key factor in the development of NAFLD among the lean Indian patients. Thus NAFLD not only confined in the obese subjects, the lean subjects also vulnerable to the development of this disorder [20].

From the present data, it may conclude that a high proportion $(\sim 47 \%)$ of the IGT subjects has NAFLD and the distribution of the disorder is almost similar in various degrees of insulin resistance in IGT subjects. Insulin resistance seems to be an independent mediator of the association between NAFLD and IGT. The data also indicate that the insulin resistance and NAFLD are associated with each other and those, in turn, are affected by adiposity in these subjects. Prospective studies with larger cohort are warranted to evaluate the markers of insulinemic indices as a screening tool in the diagnosis of NAFLD among the Bangladeshi population.

Our study had several limitations. The diagnosis of NAFLD was based on ultrasonography and was not confirmed by liver biopsy. Correlation between the different stages of NAFLD (by the histologic picture) and the levels of insulin resistance could not be done. Studies in prediabetes with different subgroups cohorts having NAFLD are needed to re-formulate the association of insulin resistance with NAFLD. Prospective studies with appropriate design should be undertaken to investigate the underlying association between HOMAIR and NAFLD in IGT subjects. 
Citation: Israt AH, Liaquat A (2016) Nonalcoholic Fatty Liver Disease and its association with Insulin Resistance: A Study from Bangladeshi Newly Diagnosed Impaired Glucose Tolerance Subjects. J Diabetes Metab 7: 688. doi: 10.4172/2155-6156.1000688

Page 5 of 5

\section{Acknowledgements}

This work was partially supported by grants from the Bangladesh Medical Research Council and Government of the People's Republic of Bangladesh, Ministry of Science and Technology (No. 39.012.002.02.01.016.2013-335).

\section{Conflict of Interest}

The authors declare that there is no conflict of interest that could be perceived as prejudicing the impartiality of the research reported.

\section{References}

1. Angulo P (2002) Nonalcoholic fatty liver disease. N Engl J Med 346: 12211231.

2. Angulo $P$ (2007) Gl epidemiology: nonalcoholic fatty liver disease. Aliment Pharmacol Ther 25: 883-889.

3. Day CP, James OF (1998) Steatohepatitis: a tale of two "hits"? Gastroenterology 114: 842-845

4. Utzschneider KM, Kahn SE (2006) Review: The role of insulin resistance in nonalcoholic fatty liver disease. J Clin Endocrinol Metab 91: 4753-4761.

5. Bugianesi E, Gastaldelli A, Vanni E, Gambino R, Cassader M, et al. (2005) Insulin resistance in non-diabetic patients with non-alcoholic fatty liver disease: sites and mechanisms. Diabetologia 48: 634-642.

6. Das K, Mukherjee PS, Ghosh A, Ghosh S, et al. (2010) Nonobese population in a developing country has a high prevalence of nonalcoholic fatty liver and significant liver disease. Hepatology 51: 1593-1602.

7. World Health Organization (2006) Definition and diagnosis of diabetes mellitus and intermediate hyperglycaemia: Report of a WHO/IDF Consultation. Geneva 25: $1151-1156$

8. Friedewald WT, Levy RI, Fredrickson DS (1972) Estimation of the concentration of low-density lipoprotein cholesterol in plasma, without use of the preparative ultracentrifuge. Clin Chem 18: 499-502.

9. Matthews DR, Hosker JP, Rudenski AS, Naylor BA, Treacher DF, et al. (1985) Homeostasis model assessment: insulin resistance and beta-cell function from fasting plasma glucose and insulin concentrations in man. Diabetologia 28 : 412-419.
10. Saadeh S, Younossi ZM, Remer EM, Gramlich T, Ong JP, et al. (2002) The utility of radiological imaging in nonalcoholic fatty liver disease. Gastroenterology 123: $745-750$.

11. Mohan V, Farooq S, Deepa M, Ravikumar R, Pitchumoni CS (2009) Prevalence of non-alcoholic fatty liver disease in urban south Indians in relation to different grades of glucose intolerance and metabolic syndrome. Diab Res and Clin Prac 84: 84-91.

12. Petersen KF, Dufour S, Feng J, Befroy D, Dziura J, et al. (2006) Increased prevalence of insulin resistance and nonalcoholic fatty liver disease in AsianIndian men. Proc Natl Acad Sci U S A 103: 18273-18277.

13. Park SH, Kim BI, Yun JW, Kim JW, Park DI, et al. (2004) Insulin resistance and $\mathrm{C}$-reactive protein as independent risk factors for non-alcoholic fatty liver disease in non-obese Asian men. J Gastroenterol Hepatol 19: 694-698.

14. Ha Y, Seo N, Shim JH, Kim SY, Park JA, et al. (2015) Intimate association of visceral obesity with non-alcoholic fatty liver disease in healthy Asians: A casecontrol study. J Gastroenterol Hepatol 30: 1666-1672.

15. Manchanayake J, Chitturi S, Nolan C, Farrell GC (2011) Postprandia hyperinsulinemia is universal in non-diabetic patients with nonalcoholic fatty liver disease. J Gastroenterol Hepatol 26: 510-516.

16. Amarapurkar D, Das HS (2002) Chronic liver disease in diabetes mellitus. Trop Gastroenterol 23: 3-5.

17. Targher G, Bertolini L, Padovani R, Poli F, Scala L, et al. (2006) Increased prevalence of cardiovascular disease in Type 2 diabetic patients with nonalcoholic fatty liver disease. Diabet Med 23: 403-409.

18. Das S, Singh SP, Parida PK, Mallick RN (2010) Nonalcoholic fatty live disease in subjects with type 2 diabetes mellitus and non-diabetics with special reference to insulin resistance and hepatic histopathological changes. Diab Metab Syndr: Clin Res Rev 226: 226-229.

19. Ortiz-Lopez C, Lomonaco R, Orsak B, Finch J, Chang Z, et al. (2012 Prevalence of prediabetes and diabetes and metabolic profile of patients with nonalcoholic fatty liver disease (NAFLD). Diabetes Care 35: 873-878.

20. Bhat G, Baba CS, Pandey A, Kumari N, Choudhuri G (2013) Insulin resistance and metabolic syndrome in nonobese Indian patients with non-alcoholic fatty liver disease. Trop Gastroenterol 34:18-24. 